\title{
$p$-TH POWER TOTAL VARIATION REGULARIZATION IN PHOTON-LIMITED IMAGING VIA ITERATIVE REWEIGHTING
}

\author{
Lasith Adhikari and Roummel F. Marcia
}

\author{
Department of Applied Mathematics, University of California, Merced, Merced, CA 95343 USA
}

\begin{abstract}
Recent work in $\ell_{p}$-norm regularized sparsity recovery problems (where $0 \leq p<1$ ) has shown that signals can be recovered with very high accuracy despite the fact that the solution to these nonconvex optimization problems are not necessarily the global minima but are instead potentially local minima. In particular, $\ell_{p}$-norm regularization has been used effectively for signal reconstruction from measurements corrupted by zero-mean additive Gaussian noise. This paper describes a $p$-th power total variation $\left(\mathrm{TV}_{p}\right)$ regularized optimization approach for image recovery problems in photonlimited settings using iterative reweighting. The proposed method iteratively convexifies a sequence of nonconvex $\mathrm{TV}_{p}$ subproblems using a weighted TV approach and is solved using a modification to the FISTA method for TV-based denoising. We explore the effectiveness of the proposed method through numerical experiments in image deblurring.
\end{abstract}

Index Terms - Total variation (TV), weighted TV, SPIRAL-TV $_{p}$, photon-limited imaging, Poisson noise

\section{INTRODUCTION}

In photon-limited imaging regimes, the number of photons received at the camera lens is relatively low. Such situations occur in nuclear medicine [1], night vision, and astronomy $[2,3]$. Data in these applications are collected by counting discrete independent events and are accurately modeled using a Poisson noise model [4]:

$$
\mathbf{y} \sim \operatorname{Poisson}\left(\mathbf{A} \mathbf{f}^{*}\right)
$$

where $\mathbf{f}^{*} \in \mathbb{R}_{+}^{n}$ is the true signal or image of interest, $\mathbf{A} \in$ $\mathbb{R}_{+}^{m \times n}$ is the linear projection matrix, and $\mathbf{y} \in \mathbb{Z}_{+}^{m}$ is a vector of observed photon counts.

Under the Poisson model, an unknown signal $\mathbf{f}^{*}$ is estimated by minimizing the negative Poisson log-likelihood function which is derived using the maximum likelihood principle. This inverse minimization problem is ill-posed if the dimension of the measurement $\mathbf{y}$ is smaller than the dimension of the unknown signal $\mathbf{f}^{*}$. Therefore, various penalization schemes are usually incorporated to pose the problem better.

This work was supported by National Science Foundation Grant CMMI1333326.
When the signal to be reconstructed is known to be sparse in the canonical basis, it can be recovered accurately using a nonconvex $\ell_{p}$-norm regularization technique [5]. However, when the image is not sparse, a different regularization must be employed. The total variation (TV) seminorm penalty [6] has been commonly used as a sparsity measure and has been shown to be very effective as a regularization term for image reconstruction. More specifically, the TV seminorm measures the first-order difference between adjacent pixels in images. Thus, an image with a small TV seminorm means that generally, it has homogeneous signal levels with few abrupt changes or edges. The recent work of Yan and Lu [7] generalizes this TV norm to the $p$-th power $\left(\mathrm{TV}_{p}\right)$, where $0 \leq p \leq 1$, by using a weighted TV minimization where the weights are computed to approximate the $\mathrm{TV}_{p}$ regularized problem locally.

In this paper, we propose to regularize the negative Poisson log-likelihood function using the $\mathrm{TV}_{p}$ penalization method in [7]. Specifically, we use the SPIRAL approach [8] to define a sequence of minimization subproblems with the $\mathrm{TV}_{p}$ penalty. These subproblems are solved using the FISTA TV-based denoising method [9]. We explore the effectiveness of the proposed method through numerical experiments in image deblurring.

\section{PROBLEM FORMULATION}

\subsection{Sparse Poisson Intensity Reconstruction}

Under the inhomogeneous Poisson process model, the Poisson intensity reconstruction problem has the following constrained minimization form:

$$
\begin{array}{ll}
\underset{\mathbf{f} \in \mathbb{R}^{n}}{\operatorname{minimize}} & \Phi(\mathbf{f}) \equiv F(\mathbf{f})+\tau \operatorname{pen}(\mathbf{f}) \\
\text { subject to } & \mathbf{f} \succeq 0,
\end{array}
$$

where $\tau>0, F(\mathbf{f})$ is the negative Poisson log-likelihood function

$$
F(\mathbf{f})=\mathbf{1}^{T} \mathbf{A f}-\sum_{i=1}^{m} y_{i} \log \left(\mathbf{e}_{i}^{T} \mathbf{A f}+\beta\right),
$$

where $\mathbf{1}$ is an $m$-vector of ones, $\mathbf{e}_{i}$ is the $i$ th canonical basis unit vector, $\beta>0$ (typically $\beta \ll 1$ ) and pen : $\mathbb{R}^{n} \longrightarrow \mathbb{R}$ is a penalty function (see e.g., [10]). 
This minimization problem can be solved by minimizing a sequence of easier subproblems. In [8], $F(\mathbf{f})$ is approximated by second-order Taylor series expansion, where the Hessian in the Taylor series is replaced by a scaled identity matrix $\alpha_{k} \mathbf{I}$, where $\alpha_{k}>0$ (see e.g., [11,12]). A simple manipulation to this quadratic approximation will lead into a sequence of subproblems of the form

$$
\begin{aligned}
\mathbf{f}^{k+1}=\underset{\substack{\mathbf{f} \in \mathbb{R}^{n} \\
\text { subject to }}}{\arg \operatorname{f} \min } \frac{1}{2}\left\|\mathbf{f}-\mathbf{s}^{k}\right\|_{2}^{2}+\frac{\tau}{\alpha_{k}} \operatorname{pen}(\mathbf{f}) \\
\text { subje }
\end{aligned}
$$

where

$$
\mathbf{s}^{k}=\mathbf{f}^{k}-\frac{1}{\alpha_{k}} \nabla F\left(\mathbf{f}^{k}\right)
$$

(see [8] for details)

\subsection{Total Variation Regularization}

The total variation seminorm is often used to promote sparsity in the gradient of an image, meaning the reconstructed image has very few abrupt changes in adjacent pixel intensities. The penalty function pen(f) in (2) can thus be replaced by $\|\mathbf{f}\|_{\mathrm{TV}_{1}}$ and the subproblem can be rewritten of the form

$$
\begin{aligned}
\mathbf{f}^{k+1}=\underset{\substack{\mathbf{f} \in \mathbb{R}^{m n} \\
\text { subject to }}}{\arg \operatorname{f} \succeq 0,} & \frac{1}{2}\left\|\mathbf{f}-\mathbf{s}^{k}\right\|_{2}^{2}+\frac{\tau}{\alpha_{k}}\|\mathbf{f}\|_{\mathrm{TV}_{1}} \\
&
\end{aligned}
$$

If $\mathbf{f} \in \mathbb{R}_{+}^{m n}$ is a $m \times n$ image, the anisotropic TV seminorm of $\mathbf{f}$ is given by

$$
\|\mathbf{f}\|_{\mathrm{TV}_{1}^{(\mathrm{A})}}=\sum_{i=1}^{m-1} \sum_{j=1}^{n}\left|f_{i, j}-f_{i+1, j}\right|+\sum_{i=1}^{m} \sum_{j=1}^{n-1}\left|f_{i, j}-f_{i, j+1}\right|,
$$

while the isotropic TV seminorm of $\mathbf{f}$ is

$$
\begin{aligned}
\|\mathbf{f}\|_{\mathrm{TV}_{1}^{(\mathrm{I})}}= & \sum_{i=1}^{m-1} \sum_{j=1}^{n-1} \sqrt{\left(f_{i, j}-f_{i+1, j}\right)^{2}+\left(f_{i, j}-f_{i, j+1}\right)^{2}} \\
& +\sum_{i=1}^{m-1}\left|f_{i, n}-f_{i+1, n}\right|+\sum_{j=1}^{n-1}\left|f_{m, j}-f_{m, j+1}\right| .
\end{aligned}
$$

In [8], the TV-based nonnegative denoising subproblem (3) is solved using the fast gradient projection FISTA algorithm [9].

\section{3. $p$-th Power Total Variation Regularization using It- erative Reweighting}

We now propose to regularize the negative Poisson log likelihood function in (1) using a $p$-th power total variation [7], denoted by $\operatorname{TV}_{p}(0 \leq p \leq 1)$. Then the sequence of subproblems has the following form

$$
\begin{aligned}
\mathbf{f}^{k+1}=\underset{\substack{\mathbf{f} \in \mathbb{R}^{m n} \\
\text { subject to }}}{\arg \operatorname{f} \succeq 0,} & \frac{1}{2}\left\|\mathbf{f}-\mathbf{s}^{k}\right\|_{2}^{2}+\frac{\tau}{\alpha_{k}}\|\mathbf{f}\|_{\mathrm{TV}_{p}} \\
&
\end{aligned}
$$

where $\|\mathbf{f}\|_{\mathrm{TV}_{p}}$ is defined as the anisotropic TV seminorm

$$
\|\mathbf{f}\|_{\mathrm{TV}_{p}^{(\mathrm{A})}}=\sum_{i=1}^{m-1} \sum_{j=1}^{n}\left|f_{i, j}-f_{i+1, j}\right|^{p}+\sum_{i=1}^{m} \sum_{j=1}^{n-1}\left|f_{i, j}-f_{i, j+1}\right|^{p},
$$

or as the isotropic TV seminorm

$$
\begin{aligned}
\|\mathbf{f}\|_{\mathbf{T V}_{p}^{(\mathrm{I})}}= & \sum_{i=1}^{m-1} \sum_{j=1}^{n-1} \sqrt{\left(f_{i, j}-f_{i+1, j}\right)^{2 p}+\left(f_{i, j}-f_{i, j+1}\right)^{2 p}} \\
& +\sum_{i=1}^{m-1}\left|f_{i, n}-f_{i+1, n}\right|^{p}+\sum_{j=1}^{n-1}\left|f_{m, j}-f_{m, j+1}\right|^{p} .
\end{aligned}
$$

Note that when $p<1$, both $\mathrm{TV}_{p}$ penalty functions are nonconvex, making the global minimum of (4) difficult to trace. In [7], the minimization of the nonconvex problem (4) is accomplished through the convexification of the nonconvex penalty function $\|\mathbf{f}\|_{\mathrm{TV}_{p}}$ using a reweighting strategy. More specifically, a weighted TV seminorm is used to approximate the anisotropic TV seminorm

$$
\begin{aligned}
\|\mathbf{f}\|_{\mathbf{T V}_{w}^{(\mathrm{A})}}= & \sum_{i=1}^{m-1} \sum_{j=1}^{n} \alpha_{i, j}\left|f_{i, j}-f_{i+1, j}\right| \\
& +\sum_{i=1}^{m} \sum_{j=1}^{n-1} \beta_{i, j}\left|f_{i, j}-f_{i, j+1}\right|,
\end{aligned}
$$

as well as the isotropic TV seminorm

$$
\begin{aligned}
& \|\mathbf{f}\|_{\mathbf{T V}_{w}^{(\mathrm{I})}}= \\
& \sum_{i=1}^{m-1} \sum_{j=1}^{n-1} \sqrt{\left(\alpha_{i, j}\left(f_{i, j}-f_{i+1, j}\right)\right)^{2}+\left(\beta_{i, j}\left(f_{i, j}-f_{i, j+1}\right)\right)^{2}} \\
& \quad+\sum_{i=1}^{m-1} \alpha_{i, n}\left|f_{i, n}-f_{i+1, n}\right|+\sum_{j=1}^{n-1} \beta_{m, j}\left|f_{m, j}-f_{m, j+1}\right| .
\end{aligned}
$$

In the above weighted TV definitions, $\alpha_{i, j}>0$ and $\beta_{i, j}>$ 0 are coefficients whose values are computed using $\mathbf{f}$ in the previous iterate $k$ :

$$
\begin{aligned}
& \alpha_{i, j}=\left(\left|f_{i, j}^{(k)}-f_{i+1, j}^{(k)}\right|+\epsilon\right)^{(p-1)}, \\
& \beta_{i, j}=\left(\left|f_{i, j}^{(k)}-f_{i, j+1}^{(k)}\right|+\epsilon\right)^{(p-1)},
\end{aligned}
$$

where $\epsilon>0$ to prevent the weights from being arbitrary large. By substituting (5) and (6) for $\alpha_{i, j}$ and $\beta_{i, j}$ respectively in the weighted TV definitions, it can be shown that $\|\mathbf{f}\|_{\mathrm{TV}_{w}^{(\mathrm{A})}} \approx$ $\left\|\mathbf{f}^{(k)}\right\|_{\mathrm{TV}_{p}^{(\mathrm{A})}}$ and $\|\mathbf{f}\|_{\mathbf{T V}_{w}^{(\mathrm{I})}} \approx\left\|\mathbf{f}^{(k)}\right\|_{\mathrm{TV}_{p}^{(\mathrm{I})}}$ (see [7] for details).

\subsection{Modified Dual Approach with Weighted TV Norms}

Using the weighted TV functions as defined in the Section 2.3, we now show how to modify the FISTA approach of dual problem construction $[9,13]$. In particular, we use the exact 
same set of notations used in [9] (Sec. 4.1) with the exception of the linear operator $\mathscr{L}$.

Let $\mathcal{P}_{A}$ be the set of matrix-pairs $\left(\mathbf{p}_{1}, \mathbf{q}_{1}\right)$ where $\mathbf{p}_{1} \in$ $\mathbb{R}^{(m-1) \times n}$ and $\mathbf{q}_{1} \in \mathbb{R}^{m \times(n-1)}$ satisfying

$$
\begin{aligned}
\left|p_{i, j}\right| \leq 1, \quad i=1, \ldots, m-1, j=1, \ldots, n \\
\left|q_{i, j}\right| \leq 1, \quad i=1, \ldots, m, j=1, \ldots, n-1
\end{aligned}
$$

Similarly, let $\mathcal{P}_{I}$ be the set of matrix-pairs $\left(\mathbf{p}_{2}, \mathbf{q}_{2}\right)$ where $\mathbf{p}_{2} \in \mathbb{R}^{(m-1) \times n}$ and $\mathbf{q}_{2} \in \mathbb{R}^{m \times(n-1)}$ that satisfy

$$
\begin{aligned}
p_{i, j}^{2}+q_{i, j}^{2} & \leq 1, \quad i=1, \ldots, m-1, j=1, \ldots, n-1 \\
\left|p_{i, n}\right| & \leq 1, \quad i=1, \ldots, m-1 \\
\left|q_{m, j}\right| & \leq 1, \quad j=1, \ldots, n-1
\end{aligned}
$$

We redefine the linear operator $\mathscr{L}: \mathbb{R}^{(m-1) \times n} \times \mathbb{R}^{m \times(n-1)} \longrightarrow$ $\mathbb{R}^{m \times n}$ as follows:

$$
\mathscr{L}\left(\mathbf{p}_{\ell}, \mathbf{q}_{\ell}\right)_{i, j}=\alpha_{i, j} p_{i, j}-\alpha_{i-1, j} p_{i-1, j}+\beta_{i, j} q_{i, j}-\beta_{i, j-1} q_{i, j-1},
$$

where $\ell$ is 1 or 2 , and we assume that $p_{0, j}=p_{m, j}=q_{i, 0}=$ $q_{i, n} \equiv 0$ for $i=1, \ldots, m, j=1, \ldots, n$. The operator $\mathscr{L}^{T}: \mathbb{R}^{m \times n} \longrightarrow \mathbb{R}^{(m-1) \times n} \times \mathbb{R}^{m \times(n-1)}$ is given by $\mathscr{L}^{T}(\mathbf{f})=\left(\mathbf{p}_{\ell}, \mathbf{q}_{\ell}\right)$, where $p_{i, j}=f_{i, j}-f_{i+1, j}$ and $q_{i, j}=$ $f_{i, j}-f_{i, j+1}$. Since our subproblems (4) are non-negatively constrained, $P_{C}$ is the orthogonal projection operator on to the set $C=[0, \infty)$.

Now note that the following two relations

$$
\begin{aligned}
\alpha|x| & =\max _{p}\{\alpha x p:|p| \leq 1\} \\
\sqrt{\alpha^{2} x^{2}+\beta^{2} y^{2}} & =\max _{p_{1}, p_{2}}\left\{\alpha x p_{1}+\beta y p_{2}: p_{1}^{2}+p_{2}^{2} \leq 1\right\},
\end{aligned}
$$

hold true for weights $\alpha>0$ and $\beta>0$. Therefore, the anisotropic weighted TV seminorm can be written as the maximization problem

$$
\|\mathbf{f}\|_{\mathrm{TV}_{w}^{(\mathrm{A})}}=\max _{\left(\mathbf{p}_{1}, \mathbf{q}_{1}\right) \in \mathcal{P}_{A}} T_{1}\left(\mathbf{f}, \mathbf{p}_{1}, \mathbf{q}_{1}\right)
$$

where

$$
\begin{aligned}
T_{1}\left(\mathbf{f}, \mathbf{p}_{1}, \mathbf{q}_{1}\right)= & \sum_{i=1}^{m-1} \sum_{j=1}^{n} \alpha_{i, j}\left(f_{i, j}-f_{i+1, j}\right) p_{i, j} \\
& +\sum_{i=1}^{m} \sum_{j=1}^{n-1} \beta_{i, j}\left(f_{i, j}-f_{i, j+1}\right) q_{i, j} .
\end{aligned}
$$

Similarly, the isotropic weighted TV seminorm also can be written as the maximization problem

$$
\|\mathbf{f}\|_{\mathrm{TV}_{w}^{(\mathrm{I})}}=\max _{\left(\mathbf{p}_{2}, \mathbf{q}_{2}\right) \in \mathcal{P}_{I}} T_{2}\left(\mathbf{f}, \mathbf{p}_{2}, \mathbf{q}_{2}\right)
$$

where

$$
\begin{aligned}
T_{2}\left(\mathbf{f}, \mathbf{p}_{2}, \mathbf{q}_{2}\right)= & \sum_{i=1}^{m-1} \sum_{j=1}^{n-1}\left[\alpha_{i, j}\left(f_{i, j}-f_{i+1, j}\right) p_{i, j}\right. \\
& \left.+\beta_{i, j}\left(f_{i, j}-f_{i, j+1}\right) q_{i, j}\right] \\
& +\sum_{i=1}^{m-1} \alpha_{i, n}\left(f_{i, n}-f_{i+1, n}\right) p_{i, n} \\
& +\sum_{j=1}^{n-1} \beta_{m, j}\left(f_{m, j}-f_{m, j+1}\right) q_{m, j} .
\end{aligned}
$$

With the above defined notations, we have

$$
\begin{aligned}
& T_{1}\left(\mathbf{f}, \mathbf{p}_{1}, \mathbf{q}_{1}\right)=\operatorname{Tr}\left(\mathscr{L}\left(\mathbf{p}_{1}, \mathbf{q}_{1}\right)^{T} \mathbf{f}\right), \\
& T_{2}\left(\mathbf{f}, \mathbf{p}_{2}, \mathbf{q}_{2}\right)=\operatorname{Tr}\left(\mathscr{L}\left(\mathbf{p}_{2}, \mathbf{q}_{2}\right)^{T} \mathbf{f}\right) .
\end{aligned}
$$

Hereafter we can follow the same procedure explained in [9] to obtain the dual problem with the weighted TV norm. This dual problem is iteratively solved using a fast gradient projection method (see [9] for more details).

\section{NUMERICAL EXPERIMENTS}

In this section, we demonstrate the effectiveness of the pro-

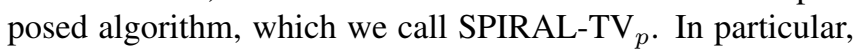
we consider an image deblurring problem for which TV norm regularization is highly suitable.

In this experimental setup, we use the Shepp-Logan phantom image of size $128 \times 128$ available in the MATLAB's image processing toolbox as the true image $\mathrm{f}^{*}$ (see Fig. 1(a)). The true detector blurred image (see Fig. 1(b)) was obtained by $\mathbf{A} \mathbf{f}^{*}$, where $\mathbf{A}$ is a blurring operator ( $\mathbf{f}^{*}$ is convolved with some blur matrix). Finally, a Poisson noisy observation matrix of size $128 \times 128$ is simulated by MATLAB's poissrnd function. The Poisson noisy observation matrix is shown in Fig. 1(b), where the mean photon count is 45.8 with a maximum of 398 .

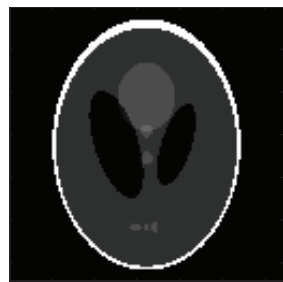

(a) Truth image (f*)

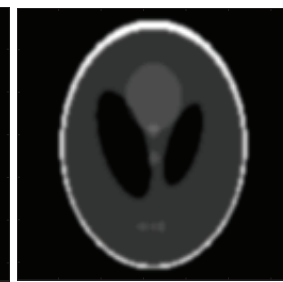

(b) True detector intensity (Af*)

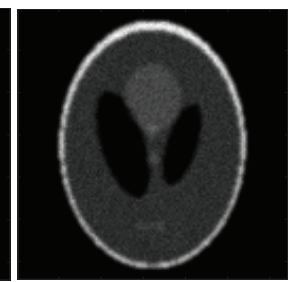

(c) Observed photon counts (y)
Fig. 1. Experimental setup: (a) Shepp-Logan head phantom as true image, (b) blurred phantom image, (c) Poisson noisy phantom image with mean count 45.8 .

We implemented the SPIRAL-TV $p$ algorithm in MATLAB R2013a (on a PC with Intel Core i7 2.7GHz Processor with 8GB memory) by modifying existing codes of the SPIRAL method [14] and the TV-based FISTA denoising method 


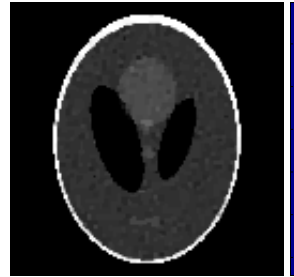

(a) SPIRAL-TV $\mathrm{PSNR}=30.70 \mathrm{~dB}$

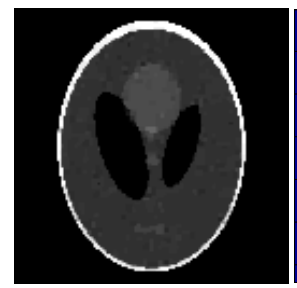

(d) SPIRAL-TV 0.8 PSNR $=30.95 \mathrm{~dB}$

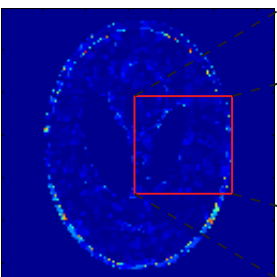

(b) Truth - SPIRAL-TV RMSE $=11.90 \%$

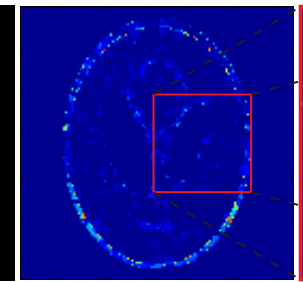

(e) Truth - SPIRAL-TV $\mathrm{RMSE}=11.57 \%$

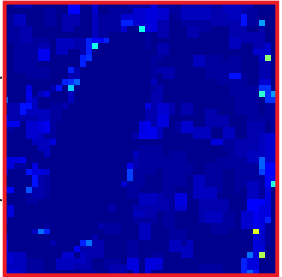

(c) Zoomed region

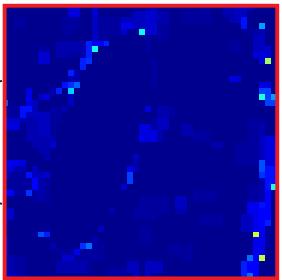

(f) Zoomed region

Fig. 2. Anisotropic TV based reconstructions and error images. Top row: (a) SPIRAL-TV ${ }_{1}$ reconstruction, (b) magnitude of error between the true image and the SPIRAL-TV estimated image, (c) SPIRAL-TV 1 reconstruction has more artifacts. Bottom row: (d) SPIRAL-TV 0.8 reconstruction, (e) magnitude of error between the true image and the SPIRAL$\mathrm{TV}_{0.8}$ estimated image, (f) SPIRAL- $\mathrm{TV}_{0.8}$ reconstruction has more homogeneous signal levels.

[15]. SPIRAL-TV ${ }_{p}$ follows a warm-start strategy, where we start the method by solving the $p=1$ case first, and then using its solution to initiate the next problem with a smaller $p$ value, say, $p=0.9$. (This can be viewed as a homotopy or continuation method.) In Eqs. (5) and (6), $\epsilon=10^{-13}$ in our numerical experiments. We run the algorithm until the relative difference between consecutive iterates converged to $\left\|\mathbf{f}^{k+1}-\mathbf{f}^{k}\right\|_{2} /\left\|\mathbf{f}^{k}\right\|_{2} \leq 10^{-8}$ with a minimum of 50 iterations. For each $p$-value, the regularization parameter in (4) is optimized to get minimum RMSE value. Finally, we compared SPIRAL-TV reconstruction with SPIRAL-TV $_{1}$ reconstruction for both isotropic and anisotropic TV types using RMSE values and peak signal-to-noise ratios (PSNR $(\mathrm{dB})=$ $\left.10 \log _{10}\left(\max \left(\mathbf{f}^{*}\right)^{2} / \mathrm{MSE}\right)\right)$

\section{RESULTS}

The results of the experiments described in the Section 3 for anisotropic and isotropic TV regularization are presented in Figs. 2 and 3 respectively. For the anisotropic TV regularization, the SPIRAL-TV $\mathrm{TV}_{0.8}$ reconstruction has RMSE $=11.57 \%$ and PSNR $=30.95 \mathrm{~dB}$ while the reconstruction for SPIRAL$\mathrm{TV}_{1}$ reconstruction has $\mathrm{RMSE}=11.90 \%$ and $\mathrm{PSNR}=30.70$ $\mathrm{dB}$. For the isotropic TV regularization, the SPIRAL-TV 0.8 reconstruction has $\mathrm{RMSE}=14.45 \%$ and PSNR $=29.02 \mathrm{~dB}$ while the reconstruction for SPIRAL-TV 1 reconstruction has $\mathrm{RMSE}=14.80 \%$ and PSNR $=28.80 \mathrm{~dB}$. In both cases and

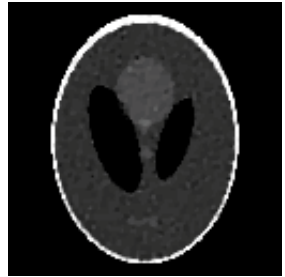

(a) SPIRAL-TV, PSNR $=28.80 \mathrm{~dB}$

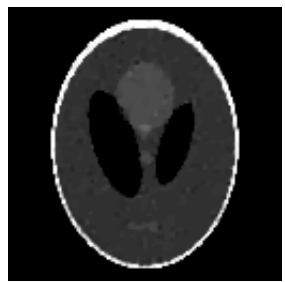

(d) SPIRAL-TV 0.8 PSNR $=29.02 \mathrm{~dB}$

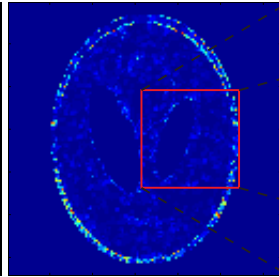

(b) Truth - SPIRAL-TV RMSE $=14.80 \%$

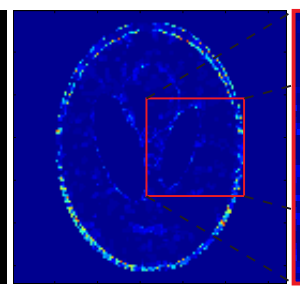

(e) Truth - SPIRAL-TV RMSE $=14.45 \%$

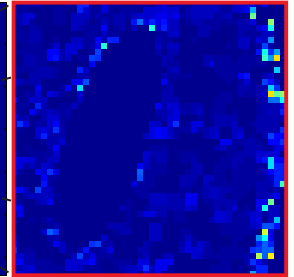

(c) Zoomed region

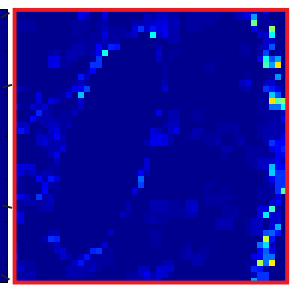

(f) Zoomed region
Fig. 3. Isotropic TV based reconstructions and error images. Top row: (a) SPIRAL-TV ${ }_{1}$ reconstruction, (b) magnitude of error between the true image and the SPIRAL-TV ${ }_{1}$ estimated image, (c) SPIRAL-TV ${ }_{1}$ reconstruction has more artifacts. Bottom row: (d) SPIRAL-TV 0.8 reconstruction, (e) magnitude of error between the true image and the SPIRAL-TV 0.8 estimated image, (f) SPIRAL-TV 0.8 reconstruction also has more homogeneous signal levels.

both metrics, the SPIRAL-TV $\mathrm{TV}_{0.8}$ reconstructions show improvement over the SPIRAL-TV 1 reconstructions. Furthermore, the SPIRAL-TV 0.8 reconstructions recovered the actual gray area in the phantom body without losing the edge details and has less prominent cloud noise-like texture (see red zoomed areas in Fig. 2 (c) and (f) and Fig. 3 (c) and (f)).

\section{DISCUSSION AND CONCLUSION}

In this paper, we have formulated a $\mathrm{TV}_{p}$ regularized negative log-likelihood function for photon-limited imaging problems. This nonconvex $\mathrm{TV}_{p}$ regularization problem is solved in a convex setting by using a reweighting strategy for each iteration. The SPIRAL-TV 1 solution is used as the warm initial point in the proposed SPIRAL-TV ${ }_{p}$ method, and we proceed with this strategy by reducing the $p$-value. Under the warm-start strategy, the proposed SPIRAL-TV ${ }_{p}$ algorithm will converge to a reasonably good local solution that is more accurate than the SPIRAL-TV 1 global solution. Since the anisotropic TV is related to the $\ell_{1}$-norm, the SPIRAL-TV with the anisotropic TV leads to more accurate results than with the isotropic TV. In our experience with this particular data set, there is no any significant improvement in reconstructions for $p$-values less than 0.8 . While the proposed SPIRAL-TV ${ }_{p}$ method leads to more accurate results with less artifacts, it requires more computational effort than SPIRAL$\mathrm{TV}_{1}$ due to the iterative nature of the warm-start strategy. 


\section{REFERENCES}

[1] J. A. Sorenson and M. E. Phelps, Physics in Nuclear Medicine, Grune \& Stratton, 1980.

[2] J. Starck and F. Murtagh, Astronomical Image and Data Analysis, Springer, 2002.

[3] J. W. Goodman and J. F. Belsher, "Fundamental limitations in linear invariant restoration of atmospherically degraded images," Proc. SPIE, vol. 0075, pp. 141-154, 1976.

[4] D. L. Snyder and M. I. Miller, "Random point processes in space and time," Springer-Verlag, New York, NY, 1991.

[5] L. Adhikari and R. F. Marcia, "Nonconvex relaxation for poisson intensity reconstruction," Accepted to 2015 IEEE International Conference on Acoustics, Speech and Signal Processing, 2015.

[6] L. I. Rudin, S. Osher, and E. Fatemi, "Nonlinear total variation based noise removal algorithms," Phys. D, vol. 60, no. 1-4, pp. 259-268, Nov. 1992.

[7] J. Yan and W. Lu, "Image denoising by generalized total variation regularization and least squares fidelity," Multidimensional Systems and Signal Processing, vol. 26, no. 1, pp. 243-266, 2015.

[8] Z. T. Harmany, R. F. Marcia, and R. M. Willett, "This is SPIRAL-TAP: Sparse Poisson intensity reconstruction algorithms; theory and practice," Image Processing, IEEE Trans. on, vol. 21, no. 3, pp. 1084-1096, March 2012.

[9] A. Beck and M. Teboulle, "Fast gradient-based algorithms for constrained total variation image denoising and deblurring problems," Image Processing, IEEE Transactions on, vol. 18, no. 11, pp. 2419-2434, Nov 2009.

[10] J. A. Fessler and A. O. Hero, "Penalized maximumlikelihood image reconstruction using space-alternating generalized em algorithms," IEEE Trans. on Image Proc., vol. 4, no. 10, pp. 1417-1429, Oct 1995.

[11] J. Barzilai and J. M. Borwein, “Two-point step size gradient methods," IMA J. Numer. Anal., vol. 8, no. 1, pp. 141-148, 1988.

[12] S. J. Wright, R. D. Nowak, and M. A. T. Figueiredo, "Sparse reconstruction by separable approximation," Signal Processing, IEEE Transactions on, vol. 57, no. 7, pp. 2479-2493, July 2009.

[13] A. Chambolle, "An algorithm for total variation minimization and applications," Journal of Mathematical Imaging and Vision, vol. 20, no. 1-2, pp. 89-97, 2004.

[14] Z. T. Harmany, R. F. Marcia, and R. M. Willett, "The Sparse Poisson Intensity Reconstruction ALgorithms (SPIRAL) Toolbox," http: / / drz.ac/ code/spiraltap/.

[15] A. Beck and M. Teboulle, "FISTA method for TV-based denoising problems," http://iew3.technion. ac.il/ becka/papers/tv_fista.zip. 